\title{
LINEAR FORMS IN FUNCTION FIELDS ${ }^{1}$
}

\section{LEONARD TORNHEIM}

We shall prove algebraically an analogue for function fields ${ }^{2}$ of a well known theorem of Minkowski on linear forms. ${ }^{3}$

Theorem 1. Let $F$ be a field and $z$ an indeterminate over $F$. Let

$$
L_{i}=\sum_{j=1}^{n} a_{i j} x_{j}, \quad i=1, \cdots, n,
$$

be $n$ linear expressions with coefficients $a_{i j}$ in $F(z)$ and with the determinant $\left|a_{i j}\right|$ of degree $d$. Then for any set of $n$ integers $c_{1}, \cdots, c_{n}$ which satisfy the condition $\sum_{i=1}^{n} c_{i}>d-n$ there exists a set of values for $x_{1}, \cdots, x_{n}$ in $F[z]$ and not all zero such that each $L_{i}$ has degree at most $c_{i}$.

First, we may assume that all of the $c_{i}$ are equal. For, suppose that $c$ is the maximum of the $c_{i}$. Write $L_{i}^{\prime}$ for $L_{i} z^{c-c_{i}}$. The determinant of the coefficients of the $L_{i}^{\prime}$ has degree $d^{\prime}=d+\sum\left(c-c_{i}\right)<\sum c+n$. If there is a set of values for $x_{1}, \cdots, x_{n}$ with the property that the degree of each $L_{i}^{\prime}$ is at most $c$, then these same values will make the degree of $L_{i}$ at most $c_{i}$.

Next, we may assume, after multiplying each $L_{i}$ by a suitable polynomial and by using an argument similar to that above, that all the $a_{i j}$ are in $F[z]$.

We shall now convert our system of $L_{i}$ by means of a transformation of determinant unity with elements in $F[z]$ into an equivalent system having $a_{i j}=0$ for $i<j$. Let $b_{1}$ be the g.c.d. of the $a_{1 j}$; then $b_{1}=\sum_{j=1}^{n} a_{1 j} c_{j 1}$ for appropriate $c_{j 1}$ in $F[z]$. Necessarily the $c_{j 1}$ are relatively prime. It is possible to find other quantities $c_{j k}(k=2, \cdots, n)$ such that the determinant $\left|c_{j k}\right|$ has value unity. ${ }^{5}$ Thus the transfor-

${ }^{1}$ Presented to the Society, April 13, 1940.

2 See M. Deuring, Zur Theorie der Idealklassen in algebraischen Funktionenkörpern, Mathematische Annalen, vol. 106 (1932), pp. 103-106, for a related result. I believe the results I prove are new.

${ }^{3} \mathrm{~A}$ bibliography of both analytic and algebraic proofs of the theorem of Minkowski on linear forms is given by $\mathrm{E}$. Jacobsthal, Der Minkowskische Linearformensatz, Sitzungsberichte Berliner mathematischen Gesellschaft, vol. 33 (1934), pp. 62-64. See also L. J. Mordell, Minkowski's theorem on homogeneous linear forms, Journal of the London Mathematical Society, vol. 8 (1933), pp. 179-192.

${ }^{4}$ The degree of a rational function is the degree of the numerator less that of the denominator. Zero is assigned the degree minus infinity.

${ }^{5}$ A. A. Albert, Normalized integral bases of algebraic number fields I, Annals of Mathematics, (2), vol. 38 (1937), p. $926 \mathrm{ff}$. The statement is proved for rational integral $c_{j k}$ but the proof applies to any integral domain having the property that a 
mation $x_{j}=\sum_{k=1}^{n} c_{j k} x_{k}^{\prime}$ has determinant unity and hence it has a reciprocal transformation with elements in $F[z]$. The forms $L_{i}$ are transformed into $L_{i}^{\prime}=\sum_{k=1}^{n} a_{i k}^{\prime} x_{k}^{\prime}$. Here $a_{1 k}^{\prime}=\sum_{j=1}^{n} a_{1 j} c_{j k}$, and, being a linear combination of $a_{1 j}$, it is divisible by their g.c.d. $b_{1} ; a_{1 k}^{\prime}=b_{1} a_{k}$. The transformation

$$
x_{1}^{\prime}=x_{1}^{\prime}-\sum_{k=2}^{n} a_{k} x_{k}^{\prime \prime}, \quad x_{r}^{\prime}=x_{r}^{\prime \prime}, \quad r=2, \cdots, n,
$$

of determinant unity transforms the $L_{i}^{\prime}$ into $L_{i}^{\prime \prime}$ with $L_{1}^{\prime \prime}=b_{1} x_{1}^{\prime \prime}$.

The procedure is repeated for the $n-1$ linear forms $M_{i}=\sum_{j=2}^{n} a_{j}^{\prime \prime} x_{j}^{\prime \prime}$ $(i=2, \cdots, n)$. Finally, if this process is continued, the resultant transformation converts the original system (1) into one with $a_{i j}=0$ for $i<j$. As a consequence, if the degree of $a_{i i}$ is $d_{i}$, then $\sum d_{i}=d$. By using another transformation of determinant unity we may assume that the degree of each $a_{i j}$ is at most $d_{i}$.

Let $G_{1}$ be the set of all $n$-tuples $\left(s_{1}, \cdots, s_{n}\right)=s$ where the $s_{i}$ are in $F[z]$ and have degree not greater than $c$; hence $G_{1}$ is a linear set over $F$ whose order $u_{1}=n(c+1)$. Write $L_{i}(s)$ for $\sum_{j=1}^{n} a_{i j} s_{j}$. Let $G_{r}$ of order $u_{r}$ over $F$ be the linear subset of $G_{1}$ composed of all quantities $s$ for which $L_{1}, \cdots, L_{r-1}$ all take values of degree not greater than $c$. Designate by $P_{r}$ the set of all $L_{r}(s)$ with $s$ in $G_{r}$, and by $Q_{r}$ the set of all polynomials in $P_{r}$ of degree not exceeding $c$. Since the maximum degree possible for a polynomial in $P_{r}$ is $c+d_{r}$, the number of linearly independent polynomials of $P_{r}$ which are not in $Q_{r}$, that is, the order of $P_{r} / Q_{r}$, is less than or equal to $d_{r}$. Now $G_{r} / G_{r+1} \simeq P_{r} / Q_{r}$, a fact which follows from the mapping of $G_{r}$ on $P_{r}$ and $G_{r+1}$ on $Q_{r}$. Hence $\left[G_{1}: G_{n+1}\right] \leqq \sum_{i=1}^{n} d_{i}=d$. Therefore the order $u_{n+1}$ of $G_{n+1}$ is not less than $n(c+1)-d$. To be sure that $G_{n+1}$ has elements other than zero, we must have $u_{n+1} \geqq 1$, that is, $n c=\sum c \geqq d+1-n$.

The following theorem applies if some of the $L_{i}$ must be made equal to zero.

THEOREM 2. If in Theorem 1 the first $m$ of the $L_{i}$ are to be made equal to zero and if their coefficients are in $F[z]$, then the conclusion will hold if $\sum_{i=m+1}^{n} c_{i}>d-(n-m)$.

For, the first $m$ polynomials $s_{i}$ must be zero if we have the transformed system used in the proof of Theorem 1. Application of Theorem 1 for the remaining $L_{i}$ yields Theorem 2 .

University of Chicago

g.c.d. of any finite number of elements exists and is linearly expressible in terms of those elements, that is, that every ideal with a finite basis is principal. 